\title{
Ecología y conservación de las aves asociadas con Polylepis: ¿qué sabemos de esta comunidad cada vez más vulnerable?
}

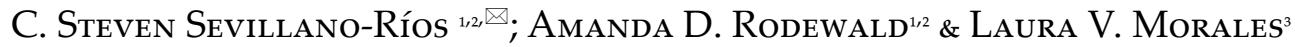 \\ ${ }^{1}$ Cornell Lab of Ornithology. Ithaca, NY, United States of America. ${ }^{2}$ Department of Natural Resources, Cornell University. \\ Ithaca, NY, United States of America. ${ }^{3}$ Department of Plant Science, University of California Davis, United States of America.
}

\begin{abstract}
Resumen. A lo largo de las montañas de Sudamérica, los bosques de Polylepis y la comunidad de aves que albergan sobresalen como uno de los ecosistemas de mayor prioridad de conservación. Estos bosques forman uno de los límites arbóreos más altos del mundo y son centros de diversidad y de endemismo biológico. Sin embargo, sus diferentes características ecológicas y las presiones de los seres humanos los hacen cada vez más vulnerables a la degradación y a los futuros cambios climáticos. Realizamos una revisión del estado del conocimiento de las diversas especies de aves asociadas con Polylepis a fin de guiar los esfuerzos para su conservación. Varias publicaciones sugieren que la función de los ecosistemas de Polylepis como hábitat para las aves está influenciada por su distribución continental y por factores locales y de paisaje, incluyendo las actividades humanas y las perturbaciones. Identificamos 29 áreas de prioridad para la conservación de Polylepis a lo largo de toda su distribución, que en conjunto mantienen 44 especies de aves endémicas, amenazadas o con alto grado de asociación con los bosques de Polylepis. Según la lista de aves amenazadas de la IUCN, dos especies están categorizadas en estado crítico, 8 en peligro, 8 vulnerables y 9 como casi amenazadas. Además, $70 \%$ de estas especies (31) poseen rangos de distribución restringida, y son endémicas a un solo país, mientras que $52 \%$ (23) poseen un alto o mediano grado de asociación con los bosques de Polylepis. Las recomendaciones de diversos estudios coinciden en que aumentar la conectividad entre parches y la permeabilidad de la matriz, y reducir la erosión, la quema y la ganadería contribuirían a mejorar la conservación y la recuperación de los ecosistemas de Polylepis y de las aves que en ella habitan.
\end{abstract}

[Palabras clave: Andes, aves andinas, cambio climático, sistemas de montaña]

\begin{abstract}
Aвstract. Ecology and conservation of Polylepis birds: what do we know about this increasingly vulnerable community? Throughout the mountains of South America, Polylepis forests and the bird community they host stand out as one of the ecosystems of greatest conservation priority. These forests form one of the highest altitudinal treelines in the world and are centers of biological diversity and endemism. However, a variety of ecological characteristics and human pressures make them increasingly vulnerable to habitat degradation and future climate change. To guide conservation efforts, we reviewed the state of knowledge for bird species associated with Polylepis forests. We describe how the Polylepis forest ecosystem's ability to support birds is influenced by its continental distribution and by local and landscape factors, including human activities and perturbations. We identified 29 priority areas for Polylepis conservation, which collectively support 44 endemic, threatened or highly specialized bird species associated with Polylepis forests. Of these, two are categorized as of critical situation, 8 as endangered, 8 as vulnerable and 9 as near threatened, according to the IUCN List of Threatened Species. In addition, $70 \%$ of these species (31) has restricted distribution ranges and are considered endemic to their country of origin, while $52 \%$ (23) has a high or medium degree of association to Polylepis forests. Recommendations from various studies agree that increasing patch connectivity and matrix permeability, and reducing soil erosion, burning and livestock stocking rates would contribute to improve the conservation and restoration of Polylepis ecosystems and the birds that inhabit it.
\end{abstract}

[Keywords: Andes, Andean birds, climate change, montane ecosystems]

\section{INTRODUCCIÓN}

Los bosques y arbustales de Polylepis son formaciones vegetales dominadas por árboles o arbustos del género Polylepis (Rosacea), restringidas a lo largo de los Andes y las Sierras de Córdoba en la Argentina (Kessler 1995; Kessler and Schmidt-Lebuhn 2006). Representan el límite arbóreo superior en el mundo (Purcell and Brelsford 2004; Hoch and Körner 2005) y se los reconoce como centros de diversidad y de endemismo de flora, fauna y de otros organismos (Fjeldså 2002; Ridbäck 2008; Gareca et al. 2010; Robledo and Renison 2010). En la actualidad, se los considera uno de los ecosistemas más amenazados de los Andes Tropicales (Herzog et al. 2011), por lo que muchos autores e instituciones expresaron la necesidad de desarrollar acciones de conservación y restauración a lo largo de toda su distribución (Fjeldså 1993; Aucca and

Editor asociado: Daniel Renison 
Ramsay 2005; Matthysen et al. 2008; Lloyd and Marsden 2008; 2011; Tinoco et al. 2013; Bellis et al. 2009; Bellis et al. 2015; Sevillano-Ríos and Rodewald 2017).

Estos bosques y las aves que los habitan fueron estudiadas en particular debido a que muchas son endémicas, categorizadas como amenazadas (IUCN 2017) o poseen un alto grado de asociación a Polylepis (Servat 2006; Lloyd 2008a,b,c). En ese sentido, creemos que es clave realizar una actualización del estado del conocimiento de las diversas especies de aves asociadas con Polylepis a lo largo de toda su distribución. Estamos convencidos de que esta actualización es muy importante dentro de un contexto de cambio climático (La Sorte and Jetz 2010) que afectaría a comunidades biológicas enteras a lo largo de los trópicos; en particular, a la avifauna de los ecosistemas de montaña que serían uno de los grupos más vulnerables (Şekercioğlu et al. 2008).

A continuación, describimos a los bosques de Polylepis en su función de hábitat para aves a través de su distribución. Primero, revisamos las asociaciones e influencias de la distribución espacial, la composición y la estructura vegetal e historia de perturbación de los bosques sobre la composición y la diversidad de aves, y destacamos lo que dichas asociaciones implican para su conservación. Segundo, resumimos el estatus de conservación actual de las especies, con el foco en las aves prioritarias para la conservación. Tercero, discutimos los factores que hacen a esta comunidad tan vulnerable al cambio climático. Finalmente, resumimos las recomendaciones a tener en cuenta al diseñar o implementar medidas de conservación, manejo y restauración de los bosques de Polylepis como hábitat para la conservación de estas aves altoandinas y las sierras de Córdoba.

\section{Los bosques de Polylepis como HÁBITAT PARA LAS AVES}

\section{La distribución espacial de Polylepis}

Los bosques de Polylepis se caracterizan por una distribución parchosa o fragmentada, tanto a escala continental (regional) como local (Simpson 1979). Esta distribución mantiene una composición de comunidades biológicas diversas, pero a la vez vulnerables a las actividades humanas (Renison et al. 2006; Renison et al. 2010) y a las anomalías ambientales que incluyen los efectos del cambio climático (Herzog et al. 2011).
Con 30 especies (Kessler and SchmidtLebuhn 2006), Polylepis se distribuye únicamente en Sudamérica a lo largo de los Andes, desde Mérida (Venezuela) hasta el norte de Chile y Argentina, con una población disyunta subtropical en las sierras de Córdoba, Argentina (Simpson 1979; Kessler 2006) (Figura 1). Aunque varias especies de Polylepis están restringidas a las partes más altas de los Andes Tropicales (3500 a 4700 m s. n. m.), hay especies que pueden estar presentes tan bajo como $900 \mathrm{~m}$ s. n. m. (e.g., P. australis), al extremo sur de su distribución, en la provincia de Córdoba, Argentina (Renison et al. 2013), o tan elevadas como 5000 m s. n. m. (e.g., P. tarapacana), en el volcán Sajama (Bolivia) (Cuyckens et al. 2016). A una escala regional, la diversidad biológica asociada con Polylepis tiende a agruparse geográficamente en lo que algunos consideran relictos de refugios pleistocénicos (Fjeldså 1992). Por lo general, estas áreas están situadas entre valles glaciares rodeados de enormes montañas y nevados que sobrepasan los $5000 \mathrm{~m} \mathrm{~s}$. n. m. (Young 2011), donde la compleja topografía habría proporcionado mayor estabilidad climática y ecológica durante largos períodos de tiempo y contribuido al desarrollo de varias especies endémicas y muy especializadas (Fjeldså et al. 1999; Fjeldså 2002; Fjeldså et al. 2012).

Sobre la base de los patrones biogeográficos de aves asociadas con Polylepis, Fjeldså (1992) reconoció tres principales centros de endemismo a lo largo de los Andes. El primero incluye a las Cordilleras Blanca, Huallanca, Huayhuash y de la Viuda, al centro norte del Perú; el segundo, al cañón del Río Apurímac y a las Cordilleras Vilcabamba y Urubamba, en Cusco; el tercero, a la Cordillera de Cochabamba, en Bolivia (Figura 1). Sin embargo, muchas otras zonas poseen agrupaciones de bosques que podrían constituir los últimos relictos de Polylepis y ser claves para la conservación de varias especies de aves, al mantener, por ejemplo, la conectividad entre poblaciones principales.

Creemos que el "Mapa Pan Andino de Bosques de Polylepis Prioritarios para la Conservación" desarrollado por Arnal et al. (2014) puede ser considerado como uno de los más precisos en cuanto a la distribución de estos bosques, que muestra una gran cantidad de áreas relictuales. Sin embargo, no pudimos encontrar un documento que explique el proceso de su elaboración (excepto por Fandiño and Caro 2009 para Colombia), $\mathrm{y}$ al inspeccionar estas áreas en imágenes 


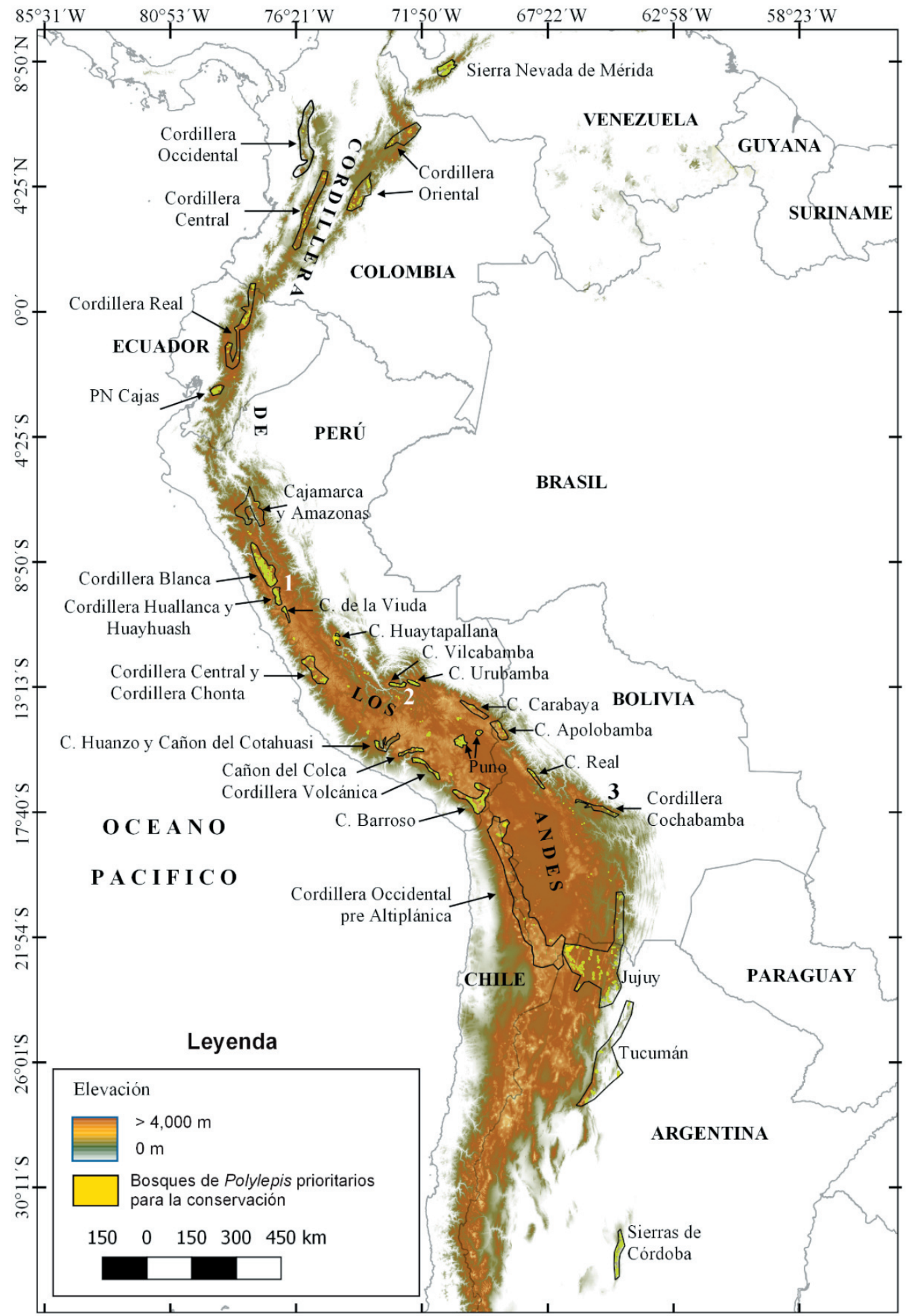

Figura 1. Distribución de los bosques de Polylepis y las áreas prioritarias para su conservación. Mostramos un mapa modificado a partir del trabajo de Arnal et al. (2014). Se incluyen los tres centros de endemismo ornitológico altoandinos sugeridos por Fjeldså (1992) (1-3) y las 29 áreas de prioridad para la conservación de Polylepis identificadas aquí.

Figure 1. Polylepis forest distribution and priority areas for its conservation. We show a modified map based on the work of Arnal et al. (2014), including the three high Andean ornithological centers of endemism suggested by Fjeldså (1992) (1-3) and the 29 priority areas for the conservation of Polylepis identified here. 
satelitales de alta resolución de Google Earth Pro $(65-50 \mathrm{~cm})$, vimos que faltaban algunas áreas con bosques o registros de Polylepis que podrían ser importantes para la avifauna. Por esto, añadimos al mapa original las localidades donde se había registrado presencia de bosques de Polylepis en otros estudios (Yallico 1992; Mendoza and Cano 2011, 2012; Valenzuela and Villalba 2015; Cuyckens et al. 2016; GBIF 2017). Identificamos y nombramos 29 áreas según las cordilleras o las localidades donde se ubicaban (Williams and Ferrigno 1999) (Figura 1). Entre éstas, destacamos algunas agrupaciones como los bosques más septentrionales, ubicados en Mérida (Venezuela), y los localizados a lo largo de las tres cordilleras Oriental, Central y Occidental en Colombia. En Ecuador, las agrupaciones principales están a lo largo de la Cordillera Real, cerca de Quito, y el Parque Nacional Cajas, cerca de Cuenca. En Perú, son interesantes los registros de Polylepis entre Cajamarca y Amazonas, que por lo general pasaron desapercibidos pese a que existen varias especies de Polylepis registradas para esta zona (Mendoza and Cano 2011, 2012; GBIF 2017), y los de los Andes de la vertiente del Pacífico entre el sur de Lima hasta Tacna (Servat 2006). Además, los bosques ubicados entre las Cordilleras de Carabaya y Apolobamba serían claves para mantener la conectividad entre Cusco y los alrededores de la Paz y Cochabamba, en Bolivia. Finalmente, destacamos los bosques y los bosquetes ubicados en ambas vertientes de los Andes entre Chile, Bolivia y Argentina, a lo largo de la Cordillera Pre-altiplánica, y el corredor entre Jujuy, Tucumán y Córdoba (Argentina).

\section{El rol de la matriz, el paisaje y la perturbación}

A escala local, las aves asociadas con Polylepis habitan principalmente en parches de diversos tamaños y formas, rodeados por una matriz de pastizal o matorral, a menudo ubicados en zonas de difícil acceso o topografía compleja. En muchos casos, estos parches están aislados de otros bosques continuos, lo cual los convierte en islas continentales. Sin embargo, aún existe el debate sobre el origen de la distribución de Polylepis (Kessler 2002; Sylvester et al. 2014), bien sea natural, mediada por microclimas favorables en un paisaje montañoso, o por una fuerte modificación humana, en particular a causa del fuego, la tala y la ganadería (Renison et al. 2006). Pese a este desacuerdo, cada vez más resultados sugieren que la distribución parchosa o fragmentada de
Polylepis sería el resultado de la interacción de factores ambientales y humanos a lo largo de miles de años (Kessler et al. 2014; Toivonen 2014; Sylvester et al. 2014; Alinari et al. 2015; Valencia et al. 2018), y se supone que la gran mayoría de las especies de aves debieron estar expuestas a estas mismas perturbaciones.

Creemos que en zonas donde Polylepis existió como un ecosistema natural parchoso por miles de años se esperaría que las aves hayan desarrollado ciertas adaptaciones para sobrevivir en este tipo de paisaje y serían menos sensibles a los cambios ambientales y espaciales, aunque no inmunes. Sin embargo, si estos bosques fueron mucho más extensos y continuos en el pasado, y la parchosidad actual fuera producto de una fragmentación más reciente, probablemente humana, se esperaría que las aves sean más sensibles a los cambios del paisaje al no estar adaptadas. En este caso, se esperaría que las poblaciones localizadas en bosques más grandes y menos aislados se desarrollen mejor que aquellas en bosques pequeños y aislados. Claro que al haber más estudios que sugieren un origen mixto de la distribución de Polylepis (Valencia et al. 2018), se esperaría más una respuesta mixta de parte de las aves, con algunas especies limitadas por la fragmentación y disponibilidad de hábitat, y otras no. Sin embargo, casi ningún estudio consideró la vulnerabilidad o la sensibilidad de las especies dentro de este marco (ver Bellis et al. 2015), que es importante por las implicaciones que tienen estos factores en la identificación de estrategias más efectivas para recuperar estas especies.

Una cantidad de estudios previos sugieren que la disponibilidad de recursos, la matriz y el grado de perturbación influyen notablemente sobre la composición y la estructura de la comunidad de aves de Polylepis (Hjarsen 1999; Herzog et al. 2003; Lloyd 2008a,c; Bellis et al. 2009; Matthysen et al. 2008; Bellis et al. 2015; Sevillano-Ríos and Rodewald 2017). Mientras que la estacionalidad (de sequía vs. lluvias) modifica en particular la disponibilidad de recursos (Herzog et al. 2003), la movilización de las aves entre parches se ve restringida por la permeabilidad y la extensión de la matriz (Lloyd and Marsden 2008). Por otro lado, las perturbaciones favorecen a que muchas de las aves de la matriz ingresen a los bosques, compitan y desplacen a las aves más especializadas, con mayores requerimientos de calidad de hábitat, pero menos versátiles. Varias aves de la matriz fueron observadas de forma regular dentro de los bosques de 
Polylepis tanto en la Argentina y Bolivia como en Perú (Herzog et al. 2003; Lloyd and Marsden 2008; Bellis et al. 2009; Sevillano-Ríos 2016). Sin embargo, otros estudios concluyen que el impacto humano en el suelo es más importante que las características estructurales de la vegetación (Bellis et al. 2015). La elevación y el grado de perturbación también pueden estar correlacionadas de manera inversa o no ser independientes, dado que el acceso a elevaciones menores permite que éstas sean fácilmente perturbadas por el hombre (Sevillano-Ríos 2010).

En conclusión, la mayoría de las recomendaciones actuales apuntan a que aumentar la conectividad entre parches y la permeabilidad de la matriz, y reducir la erosión, la quema y la ganadería serían las acciones clave para promover la regeneración natural del hábitat de las aves especialistas de Polylepis (Cierjacks et al. 2008; Torres et al. 2008; Renison et al. 2010; Domic et al. 2014; Morales 2017).

\section{AvES PRIORITARIAS PARA LA CONSERVACIÓN EN LOS BOSQUES DE Polylepis}

Basados en los criterios de endemismo (por país), categoría de amenaza (IUCN 2017) y grado de especialización a Polylepis (Fjeldså 2002; Servat 2006; Lloyd 2008a,b; Bellis et al. 2009; Tinoco et al. 2013; Sevillano-Ríos and Rodewald 2017), identificamos 44 especies de aves asociadas con al menos una de las 29 áreas antes detectadas. Estas especies deberían ser consideradas prioritarias para la conservación (Tablas 1 y 2). Para cada una de las 29 áreas también identificamos a las distintas especies de Polylepis reportadas en la bibliografía (Yallico 1992; Kessler and Schmidt-Lebuhn 2006; Fandiño and Caro 2009; Valderrama and Verhelst 2009; Mendoza and Cano 2011, 2012; Zutta et al. 2012; Renison et al. 2013; Valenzuela and Villalba 2015; Cuyckens et al. 2016; GBIF 2017; Sevillano-Ríos and Rodewald 2017) (Tabla 3).

Una de las aves de Polylepis con mayor grado de amenaza es el Churrete Real (Cinclodes aricomae) (Lloyd 2008a,b; Auca et al. 2015). El Churrete Real está categorizado como críticamente amenazado (CR) y depende casi exclusivamente de los bosques de Polylepis del sur de Perú (departamentos de Cusco, Apurímac, Ayacucho y Junín) y del noroeste de Bolivia (departamento de La Paz) (BirdLife
Tabla 1. Resumen del estatus de conservación de las especies de aves asociadas con Polylepis con prioridad para la conservación. Identificamos 44 especies prioritarias para la conservación asociadas con los bosques de Polylepis a lo largo de Sudamérica. La prioridad se basa en su grado de endemismo, su categoría de amenaza y su grado de asociación con Polylepis.

Table 1. Summary of the conservation status of Polylepis bird species of conservation priority. In total, we found 44 species of conservation priority in the Polylepis forest throughout South America. Priority was assigned based on their degree of endemism, the threat category according to the IUCN Red List 2017 and their degree of association with Polylepis.

\begin{tabular}{lc}
\hline Categorías & $\begin{array}{c}\text { Nro. de } \\
\text { especies }\end{array}$ \\
\hline Endémicas & 31 \\
Críticamente amenazadas (CR) & 2 \\
En peligro (EN) & 8 \\
Vulnerable (VU) & 8 \\
Casi amenazada (NT) & 9 \\
Fuera de peligro (LC) & 17 \\
Mediano grado de asociación con Polylepis & 18 \\
Alto grado de asociación con Polylepis & 5 \\
Total de especies & 44 \\
\hline
\end{tabular}

International 2017; Aucca et al. 2015). Según los expertos, su población, estimada entre 50-249 individuos (Tabla 2), se viene reduciendo en la mayor parte de su distribución a causa de las quemas y la ganadería intensa, que afectan la cobertura de musgos y la regeneración natural del bosque (BirdLife International 2017). Otras dos especies, el Torito de Pecho Cenizo (Anairetes alpinus) y el Tijeral de Ceja Blanca (Leptasthenura xenothorax) están categorizadas como especies en peligro (EN) y aunque sus poblaciones no han sido estimadas con rigor, los expertos sugieren que la primera rondaría entre 150 y 700 individuos adultos, y la segunda entre 250 y 999 (BirdLife International 2017). Las principales poblaciones para el Torito de Pecho Cenizo se encuentran en Cordillera Blanca, Abancay-Cusco y La Cordillera Real (La Paz, Bolivia), mientras que el Tijeral de Ceja Blanca está restringido sólo a los bosques de Polylepis de Apurímac y Cordillera Urubamba, en Cusco (Schulenberg et al. 2010). Lloyd (2008c) concluyó que en Cusco, estas especies tienen densidades poblacionales muy bajas, con menos de 5 individuos $/ \mathrm{km}^{2}$ del Churrete Real y el Torito de Pecho Cenizo, y $\sim 25$ individuos $/ \mathrm{km}^{2}$ del Tijeral de Ceja Blanca, en el mejor de los casos.

La Monterita de Cola Simple (Microspingus alticola) es otra especie en peligro(EN) (BirdLife International 2017), asociada con bosques mixtos de Polylepis y alisos (Alnus acuminata) de los departamentos de Ancash, La Libertad y Cajamarca (norte del Perú). Suele ser difícil 
Tabla 2. Lista de las especies de aves asociadas con Polylepis con prioridad para la conservación. Para cada una se muestra su rango de elevación, extensión de ocurrencia (EOO) y el tamaño poblacional (número de adultos) de acuerdo con la IUCN 2017. NA: no disponible. E: endémicas (para un país). *: medianamente especialistas. **: especialistas de Polylepis.

Table 2. List of Polylepis bird species of conservation priority. For each species, the elevational range, extent of occupancy (EOO) and population (number of adult individuals) according to the IUCN 2017 are shown. NA: not available. E: endemic (for a country). ${ }^{*}$ : moderately specialists. ${ }^{* *}$ : Polylepis specialists.

\begin{tabular}{|c|c|c|c|}
\hline Nombre de la especie & Rango de elevación (m) & $\mathrm{EOO}\left(\mathrm{km}^{2}\right)$ & Población \\
\hline Aglaeactis castelnaudii: Agcas $^{\mathrm{E}}$ (LC) & $3500-4300$ & 94300 & NA \\
\hline Anairetes alpinus: Analp $(\mathrm{EN})^{* *}$ & $3700-4500$ & 203000 & $150-700$ \\
\hline Asthenes heterura: Ashet (NT)* & $2500-4200$ & 227000 & NA \\
\hline Asthenes ottonis: Asott ${ }^{\mathrm{E}}(\mathrm{LC})$ & $2750-4000$ & 55200 & NA \\
\hline Asthenes urubambensis: Asuru (NT) & $2750-4300$ & 26200 & NA \\
\hline Asthenes virgata: Asvir ${ }^{\mathrm{E}}(\mathrm{LC})^{*}$ & $3250-4500$ & 98100 & NA \\
\hline Atlapetes canigenis: Atcan ${ }^{\mathrm{E}}$ (LC) & $2500-3000$ & 47100 & NA \\
\hline Atlapetes forbesi: Atfor ${ }^{\mathrm{E}}(\mathrm{LC})^{*}$ & $2500-3500$ & 21000 & NA \\
\hline Atlapetes rufigenis: Atruf ${ }^{\mathrm{E}}(\mathrm{NT})^{*}$ & $2750-4000$ & 22200 & NA \\
\hline Atlapetes terborghi: Atter ${ }^{\mathrm{E}}(\mathrm{NT})$ & $1700-2250$ & 5900 & NA \\
\hline Bolborhynchus ferrugineifrons: Bofer ${ }^{\mathrm{E}}$ (VU) & $3200-4000$ & 18600 & $1300-2700$ \\
\hline Cinclodes aricomae: Ciari ${ }^{\mathrm{E}}(\mathrm{CR})^{* *}$ & $3500-4800$ & 91100 & $50-249$ \\
\hline Cinclodes olrogi: Ciolr ${ }^{\mathrm{E}}(\mathrm{LC})$ & $1600-2800$ & 14800 & NA \\
\hline Coeligena orina: Coori ${ }^{\mathrm{E}}(\mathrm{CR})$ & $3150-3500$ & 140 & $50-249$ \\
\hline Conirostrum binghami: Cobin $(\mathrm{NT})^{* *}$ & $3200-4500$ & 1380000 & NA \\
\hline Conirostrum tamarugense: Cotam (VU) & $120-4100$ & 4900 & NA \\
\hline Cranioleuca albicapilla: Cralb ${ }^{\mathrm{E}}$ (LC) & $2400-3800$ & 36200 & NA \\
\hline Cranioleuca baroni: Crbar $^{\mathrm{E}}(\mathrm{LC})^{*}$ & $1700-4500$ & 110000 & NA \\
\hline Diglossa carbonaria: Dicar $^{\mathrm{E}}(\mathrm{EN})$ & $2500-4000$ & 80700 & NA \\
\hline Diglossa gloriosissima: Diglo (EN) & $3000-3800$ & 2400 & 1000-2499 \\
\hline Doliornis remseni: Dorem (VU) & $2875-3650$ & 93300 & 2500-9999 \\
\hline Eriocnemis derbyi: Erder ${ }^{\mathrm{E}}(\mathrm{NT})^{*}$ & $2500-3600$ & 39500 & NA \\
\hline Geocerthia serrana: Geser ${ }^{\mathrm{E}}(\mathrm{LC})^{*}$ & $2750-4300$ & 121000 & NA \\
\hline Leptasthenura pileata: Lepil ${ }^{\mathrm{E}}(\mathrm{LC})^{*}$ & $2000-4300$ & 104000 & NA \\
\hline Leptasthenura xenothorax: Lexen ${ }^{\mathrm{E}}(\mathrm{EN})^{* *}$ & $3700-4550$ & 7600 & $250-999$ \\
\hline Leptosittaca branickii: Lebra (VU) & $2400-3400$ & 984000 & $1500-7000$ \\
\hline Metallura phoebe: Mepho ${ }^{\mathrm{E}}(\mathrm{LC})^{*}$ & $1500-4500$ & 223000 & NA \\
\hline Microspingus alticola: Mialt ${ }^{\mathrm{E}}(\mathrm{EN})^{*}$ & $3500-4300$ & 32000 & $600-1700$ \\
\hline Oreonympha nobilis: Ornob ${ }^{\mathrm{E}}$ (LC) & $2500-3800$ & 37500 & NA \\
\hline Oreotrochilus adela: Orade (NT)* & $2550-4000$ & 95800 & $6700-6700$ \\
\hline Poospiza baeri: Pobae ${ }^{\mathrm{E}}(\mathrm{VU})$ & $2000-3150$ & 76400 & $1500-7000$ \\
\hline Poospiza garleppi: $\operatorname{Pogar}^{\mathrm{E}}(\mathrm{EN})^{*}$ & $2700-3900$ & 3800 & $270-2700$ \\
\hline Poospiza rubecula: Porub E (EN) & $2350-3700$ & 26600 & $150-700$ \\
\hline Poospizopsis caesar: Pocae ${ }^{\mathrm{E}}$ (LC) & $2500-3500$ & 32500 & NA \\
\hline Pseudosaltator rufiventris: Psruf (NT)* & $2500-4000$ & 250000 & NA \\
\hline Pyrrhura calliptera: Pycal E (VU) $^{\text {E }}$ & $1700-3400$ & 18900 & $3300-6700$ \\
\hline Scytalopus affinis: Scaff ${ }^{\mathrm{E}}(\mathrm{LC})^{*}$ & $3000-4600$ & 16000 & NA \\
\hline Scytalopus urubambae: Scuru ${ }^{\mathrm{E}}(\mathrm{LC})^{*}$ & $3500-4200$ & 14800 & NA \\
\hline Spinus crassirostris: Spcra (LC) ${ }^{*}$ & $3000-4000$ & 1750000 & NA \\
\hline Sylviorthorhynchus yanacensis: Syyan ${ }^{\mathrm{E}}(\mathrm{NT})^{*}$ & $2900-4600$ & 409000 & NA \\
\hline Taphrolesbia griseiventris: Tagri ${ }^{\mathrm{E}}(\mathrm{EN})$ & $2750-3850$ & 28000 & $250-999$ \\
\hline Tephrophilus wetmorei: Tewet (VU) & $2900-3550$ & 78200 & $1500-7000$ \\
\hline Xenodacnis parina: Xepar (LC)* & $3000-4000$ & 139000 & NA \\
\hline Zaratornis stresemanni: Zastr ${ }^{\mathrm{E}}(\mathrm{VU})^{* *}$ & $3250-4250$ & 116000 & $1000-4000$ \\
\hline
\end{tabular}

de detectar ya que está descripta como rara (Frimer and Nielsen 1989; Fjeldså and Krabbe 1990; Servat 2006) y su población posee entre 600 y 1700 individuos (BirdLife International
2017). No obstante, observaciones recientes en Ancash la muestran formando grupos familiares de 3 a 5 individuos o conformando bandadas mixtas junto con la Reinita de 
Tabla 3. Especies de aves con prioridad de conservación y especies de Polylepis registradas en las 29 áreas de prioritarias de conservación. Cada área fue nombrada principalmente según la cordillera donde se ubica. Ver la Tabla 2 para los códigos de aves. * Los nombres taxonómicos de Polylepis siguen a Schmidt-Lebuhn et al. (2006).

Table 3. Bird species of conservation priority and Polylepis species found in the 29 areas of conservation priority. Each area was named primarily according to the cordillera where it is located. See Table 2 for the bird codes. ${ }^{*}$ Polylepis scientific names follow the taxonomy of Schmidt-Lebuhn et al. (2006).

\begin{tabular}{|c|c|c|}
\hline Áreas prioritarias de Polylepis & Aves prioritarias para la conservación & Especies de Polylepis* \\
\hline Mérida (Ve) & Ninguna & P. sericea \\
\hline Cordillera Oriental (Co) & Pycal & P. quadrijuga \\
\hline Cordillera Central (Co) & Bofer, Dorem, Erder, Lebra, Tewet & P. sericea \\
\hline Cordillera Occidental (Co) & Coori, Diglo & P. cuadrijuga \\
\hline Cordillera Real de Quito (Ec) & Cobin, Dorem, Erder, Lebra, Tewet & $\begin{array}{l}P . \text { lanuginosa, } P \text {. pauta, } P \text {. sericea, } P \text {. weberbaueri, } P \text {. } \\
\text { microphylla, } P \text {. reticulata, } P \text {. incana }\end{array}$ \\
\hline Parque Nacional Cajas (Ec) & Cobin, Lebra, Xepar & $\begin{array}{l}\text { P. lanuginosa, P. sericea, } P \text {. weberbaueri, P. reticulata, } \\
P . \text { incana }\end{array}$ \\
\hline $\begin{array}{l}\text { Cajamarca, San Martín y } \\
\text { Amazonas }(\mathrm{Pe})\end{array}$ & $\begin{array}{l}\text { Atruf, Crbar, Geser, Lebra, Lepil, Mepho, } \\
\text { Mialt, Porub, Tagri, Xepar }\end{array}$ & $\begin{array}{l}\text { P. multijuga, P. weberbaueri, } P \text {. racemosa, } P \text {. } \\
\text { reticulata, P. pepei, P. pauta }\end{array}$ \\
\hline Cordillera Blanca $(\mathrm{Pe})$ & $\begin{array}{l}\text { Analp, Atruf, Crbar, Cobin, Lepil, } \\
\text { Mepho, Mialt, Geser, Porub, Spcra, Tagri, } \\
\text { Scaff, Syyan, Xepar, Zastr }\end{array}$ & $\begin{array}{l}\text { P. sericea, } P \text {. weberbaueri, } P \text {. microphylla, P. reticulata, } \\
\text { P. incana, P. racemosa }\end{array}$ \\
\hline Cordillera Huallanca (Pe) & $\begin{array}{l}\text { Analp, Crbar, Cobin, Geser, Lepil, } \\
\text { Mepho, Scaff, Spcra, Syyan, Xepar, Zastr }\end{array}$ & P. weberbaueri, $P$. reticulata, $P$. incana, $P$. racemosa \\
\hline Cordillera Huayhuash (Pe) & $\begin{array}{l}\text { Analp, Crbar, Cobin, Geser, Lepil, } \\
\text { Mepho, Scaff, Spcra, Syyan, Xepar, Zastr }\end{array}$ & $\begin{array}{l}\text { P. sericea, P. weberbaueri, P. microphylla, P. reticulata, } \\
\text { P. incana, P. racemosa }\end{array}$ \\
\hline Cordillera La Viuda (Pe) & $\begin{array}{l}\text { Analp, Cobin, Crbar, Geser, Lepil, } \\
\text { Mepho, Spcra, Xepar, Zastr }\end{array}$ & P. weberbaueri, $P$. reticulata, $P$. racemosa, $P$. flavipila \\
\hline Cordillera Huaytapallana (Pe) & Asvir, Ciari, Cralb, Lepil, Lebra, Xepar & P. rodolfo-vasquezii \\
\hline $\begin{array}{l}\text { Cordillera Central y Chonta } \\
(\mathrm{Pe})\end{array}$ & $\begin{array}{l}\text { Analp, Asvir, Cobin, Geser, Lepil, Syyan, } \\
\text { Mepho, Porub, Spcra, Zastr }\end{array}$ & $\begin{array}{l}\text { P. sericea, } P \text {. reticulata, } P \text {. incana, } P \text {. racemosa, } P \text {. } \\
\text { lanata, } P \text {. subsericans, } P \text {. flavipila }\end{array}$ \\
\hline Cordillera Vilcabamba (Pe) & $\begin{array}{l}\text { Agcas, Analp, Asott, Asuru, Asvir, Atcan, } \\
\text { Atfor, Atter, Ciari, Cralb, Cobin, Lexen, } \\
\text { Ornob, Pocae, Scuru, Spcra, Syyan, Xepar }\end{array}$ & $\begin{array}{l}\text { P. pauta, } P \text {. sericea, } P \text {. pepei, } P \text {. microphylla, } P \text {. incana, } \\
\text { P. racemosa, } P \text {. triacontandra, } P \text {. lanata, } P \text {. subsericans, } \\
P \text {. canoi }\end{array}$ \\
\hline Cordillera Urubamba (Pe) & $\begin{array}{l}\text { Agcas, Analp, Atcan, Atfor, Asott, Asuru, } \\
\text { Asvir, Ciari, Cralb, Cobin, Lexen, Ornob, } \\
\text { Pocae, Scuru, Spcra, Syyan, Xepar }\end{array}$ & $\begin{array}{l}P \text {. pauta, } P \text {. sericea, } P \text {. pepei, } P \text {. incana, } P \text {. lanata, } P \text {. } \\
\text { incarum }, P \text {. besseri, } P \text {. subsericans, } P \text {. aff besseri }\end{array}$ \\
\hline Cordillera Carabaya (Pe) & Analp, Asuru, Cobin, Syyan & P. pauta, P. pepei, P. triacontandra, P. incarum \\
\hline $\begin{array}{l}\text { Cordillera Apolobamba (Pe } \\
\text { and Bo) }\end{array}$ & Analp, Asuru, Ciari, Cobin, Spcra & $\begin{array}{l}\text { P. pauta, P. sericea, P. pepei, P. triacontandra, P. } \\
\text { besseri, P. pacensis }\end{array}$ \\
\hline Puno $(\mathrm{Pe})$ & Cobin, Spcra & $\begin{array}{l}\text { P. triacontandra, } P \text {. incarum, } P \text {. rugulosa, } P \text {. } \\
\text { pallidistigma, } P \text {. incana }\end{array}$ \\
\hline $\begin{array}{l}\text { Cordillera Huanzo y Cañón } \\
\text { del Cotahuasi }(\mathrm{Pe})\end{array}$ & Cobin, Mepho, Lepil, Spcra, Xepar & P. besseri, P. tomentella, P. microphilla \\
\hline $\begin{array}{l}\text { Cordillera del Cañón del } \\
\text { Colca }(\mathrm{Pe})\end{array}$ & Cobin, Mepho, Lepil, Spcra, Xepar & P. rugulosa \\
\hline Cordillera Volcánica (Pe) & Cobin, Cotam, Mepho, Spcra, Xepar & P. subtusalbida, P. besseri, P. rugulosa, P. tarapacana \\
\hline Cordillera Barroso (Pe) & Cobin, Cotam, Mepho, Spcra, Xepar & $\begin{array}{l}\text { P. sericea, } P \text {. incarum, } P \text {. subtusalbida, } P \text {. rugulosa, } P \text {. } \\
\text { tomentella tomentella, } P \text {. tarapacana }\end{array}$ \\
\hline Cordillera Real de la Paz (Bo) & $\begin{array}{l}\text { Agpam, Analp, Asuru, Ashet, Ciari, } \\
\text { Cobin, Dicar, Orade, Psruf, Spcra, Syyan }\end{array}$ & $\begin{array}{l}\text { P. sericea, P. pepei, P. triacontandra, P. lanata, } P \text {. } \\
\text { incarum, } P \text {. besseri, P. pacensis }\end{array}$ \\
\hline Cochabamba (Bo) & $\begin{array}{l}\text { Agpam, Asuru, Ashet, Cobin, Dicar, } \\
\text { Orade, Pogar, Psruf, Spcra, Syyan }\end{array}$ & $\begin{array}{l}P \text {. sericea, } P \text {. pepei, } P \text {. hieronymi, } P \text {. lanata, } P \text {. } \\
\text { incarum, } P \text {. subtusalbida, } P \text {. besseri, } P \text {. tomentella } \\
\text { nana, } P \text {. tomentella incanoides, } P \text {. neglecta, } P \text {. pacensis }\end{array}$ \\
\hline $\begin{array}{l}\text { Cordillera Pre-Altiplánica (Ch } \\
\text { and Bo) }\end{array}$ & Cotam, Cobin, Spcra, Syyan & P. rugulosa, P. tarapacana \\
\hline Cordillera Jujuy (Ar) & $\begin{array}{l}\text { Ashet, Cobin, Orade, Pobae, Psruf, Spcra, } \\
\text { Syyan }\end{array}$ & $\begin{array}{l}\text { P. hieronymi, P. tomentella tomentella, P. tarapacana, } \\
\text { P. crista-galli, P. australis }\end{array}$ \\
\hline Cordillera Tucumán (Ar) & Pobae, Spcra & P. hieronymi, $P$. australis \\
\hline $\begin{array}{l}\text { Cordillera Sierras de Córdoba } \\
\text { (Ar) }\end{array}$ & Ciolr, Pobae & P. australis \\
\hline
\end{tabular}


Cresta Negra (Myiothlypis nigrocristata), Tijeral colorado (Leptasthenura pileata), Cachudito Crestinegro (Anairetes nigrocristatus), el Matorralero de Oreja Rufa (Atlapetes rufigenis), Baron Cola de Espina (Cranioleuca baroni) y el Picocono Gigante o Ave del Queñual (Conirostrum binghami) - antes Oreomanes fraseri- entre otros (Sevillano-Ríos 2016; www.ebird.org). La Cotinga de Cachetes Blancos (Zaratornis stresemanni) es una especie vulnerable (VU), con una población entre 1000 a 4000 individuos adultos restringidos a los bosques de Polylepis/Gynoxys de la zona norte-centro del Perú (BirdLife International 2017). Por lo general, la distribución de esta especie está asociada con la de Tristerix spp., una Lorantacea hemi-parásita que suele crecer sobre muchos árboles de Polylepis y constituye el principal alimento de esta cotinga (Parker 1981). El Tijeral Leonado (Sylviorthorhynchus yanacensis) es una especie cercana al peligro (NT), descrita como poco común en Cordillera Blanca, en los departamentos de Apurímac y Cusco y a lo largo de los Andes del Sur, desde La Paz hasta Jujuy (Bellis et al. 2009; Schulenberg et al. 2010; BirdLife International 2017).

Otras especies de preocupación para la conservación se encuentran menos restringidas y poseen rangos de distribución relativamente amplios. El Picocono Gigante, es el ave más característica de casi cualquier bosque de Polylepis (Vuilleumier 1984; Fjeldså 2002). Esta especie está categorizada como casi amenazada (NT) por su alto grado de especialización a Polylepis (BirdLife International 2017). Se distribuye desde el sur de Colombia (Vuilleumier 1984) hasta el noroeste de la Argentina (Salta) y norte de Chile (Mazar-Barnett et al. 1998).

Por otro lado, algunas especies no especializadas a Polylepis, pero prioritarias para la conservación, también usan y dependen estos bosques. Por ejemplo, los bosques de Polylepis de Colombia también son hábitat clave para varias aves endémicas y amenazadas mundialmente como el Colibrí del Sol (Coeligena orina), el Pinchaflor Pechirrufo (Diglossa gloriosissima) y el Periquito Frentirrufo (Bolborhynchus ferrugineifrons), entre otros (Valderrama and Verhelst 2009). El Colibrí del Sol está restringido a 100 $\mathrm{km}^{2}$ en la Cordillera Occidental, con una población decreciente estimada en menos de 250 individuos. Se la considera como una especie críticamente amenazada (CR) (BirdLife International 2017). En los bosques de la Cordillera Volcánica de Perú también se registra de forma estacional al Picocono del Tamarugo (Conirostrum tamarugense), una especie clasificada como vulnerable que durante la época no reproductiva ocupa los bosques de Polylepis de esta zona (BirdLife International 2017). Finalmente, especies actualmente no reconocidas en la lista roja de especies amenazadas (IUCN 2017), como el Azulito Andino (Xenodacnis parina), el Jilguero de Pico Grueso (Spinus crassirostris), el Totoroi de Cabeza Listada (Grallaria andicolus) o el Tapaculo de Ancash (Scytalopus affinis), podrían estar en riesgo debido sus rangos de distribución restringida y a otros factores que las predisponen a la extinción (ver la siguiente sección). Estas especies requieren un monitoreo cuidadoso de sus poblaciones, en especial dentro de contexto de un cambio climático (Ocampo-Peñuela et al. 2016).

\section{Vulnerabilidad AL CAMBio CLIMÁTICO}

Los ecosistemas de Polylepis son muy vulnerables al cambio climático debido al mayor grado de anomalías ambientales que se esperan para los Andes (Urrutia and Vuille 2009) y al incremento del riesgo de extinción con la elevación (Pekercioðlu et al. 2008; La Sorte and Jetz 2010; Robledo and Renison 2010). Una de las principales evidencias de los efectos del cambio climático en los Andes es la reducción de la masa glaciar entre 20 y $30 \%$ durante los últimos 40 años (Vuille et al. 2008; Rabatel et al. 2013), atribuida principalmente al incremento de la temperatura a mayores elevaciones (Fraser 2012; Martínez et al. 2011). Por otro lado, aunque los regímenes de precipitación no muestran un patrón concluyente entre los Andes de Ecuador y Venezuela, existe una tendencia de sequía para los Andes del sur, entre Perú y Bolivia (Neelin et al. 2006; Martínez et al. 2011). Desafortunadamente, bajo condiciones semejantes, de mayor temperatura y sequía, se registraron las contracciones más severas de los bosques de Polylepis durante el pleistoceno tardío (Gosling et al. 2009) y se esperaría que tengan efectos negativos sobre las distribuciones, las interacciones y los ciclos biológicos de muchas especies (Wormworth and Mallon 2007; Møller et al. 2010; Wormworth and Şekercioğlu 2011; Şekercioğlu et al. 2012).

Una de las principales consecuencias del cambio climático será la modificación del rango de distribución altitudinal de 
diversas especies, en especial una migración a mayores elevaciones (Parmesan 2006). Sin embargo, en el caso de las aves de Polylepis esto generaría la contracción del rango de distribución de muchas de ellas dado que no podrían extenderse más allá de los límites superiores de la gradiente altitudinal. Esto generaría un número elevado de extinciones en las cimas de las montañas, algo conocido como "mountain-top extinctions" (Colwell et al. 2008). Además, incluso si los bosques de Polylepis no disminuyen, ya que demostraron ser bastante estables en algunos lugares (Jameson and Ramsay 2007), es poco probable que aumenten su distribución a mayores elevaciones (Rehm and Feeley 2013; Morales 2017). Esta estabilidad evitaría que muchas aves migren a mayores elevaciones por la falta de hábitat y que enfrenten la llegada de especies colonizadoras provenientes de menores elevaciones (Rehm and Feeley 2016).

Además, es importante reconocer que la mayoría de las especies de la Tabla 2 poseen tamaños poblacionales reducidos (Figura 2a) y rangos restringidos de distribución (Figura 2b), lo cual los predispone aun más a la extinción. Varias están distribuidas en muchas sub-poblaciones locales pequeñas, lo que eleva la probabilidad de extinciones locales para un gran número de las especies de aves asociadas con Polylepis, incluso si en la actualidad no están categorizadas como amenazadas (Crick 2004; Şekercioğlu et al. 2012; IUCN 2017).

\section{ReCOMENDACIONES}

Creemos que futuros proyectos centrados en la conservación de los bosques de Polylepis y sus comunidades de aves asociadas deberían incorporar las siguientes recomendaciones: 1) aumentar el número de áreas prioritarias para la conservación legalmente protegidas (Zutta et al. 2012) e involucrar a las poblaciones humanas locales. Los diversos mecanismos de protección, como reconocer áreas intangibles y áreas de protección nacional, regional, local, comunal o privada, deberían ser promovidos por los Estados (Aucca and Ramsay 2005), con la colaboración de comunidades locales, de ONGs y tomadores de decisiones, del sistema legal y de la academia; 2) a una escala local, las aves más vulnerables se encontrarían a mayores alturas. Por lo tanto, aunque es posible que exista un hábitat adecuado a menores elevaciones (SevillanoRíos 2016), esto no bastaría. Se debería promover la conservación y la restauración de los bosques a mayores elevaciones (Lloyd 2008b; Cahill and Matthysen 2007; Márcora et al. 2008; Tinoco et al. 2013; Sevillano-Ríos and Rodewald 2017); 3) aunque el rol de los parches pequeños de Polylepis -incluso de los árboles individuales - no es comprendido a)

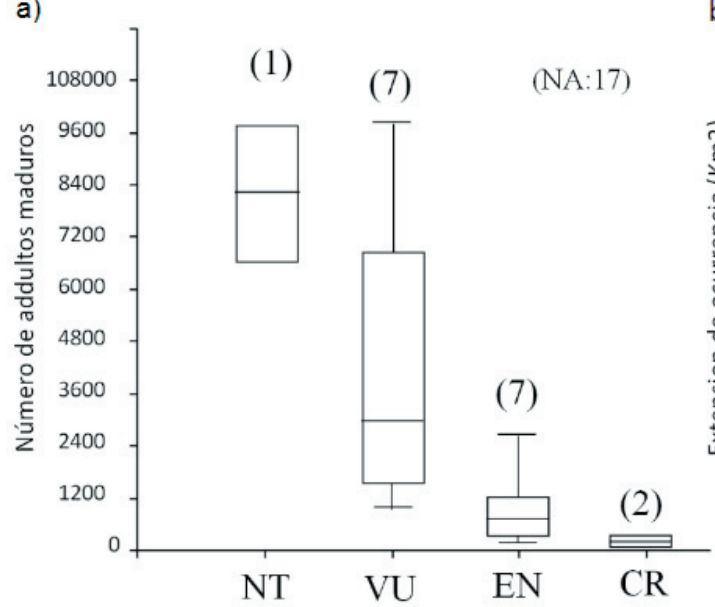

b)

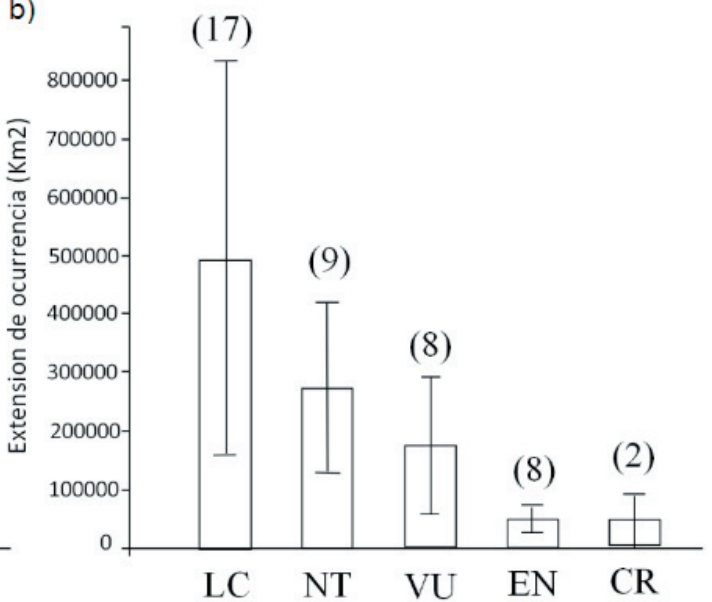

Figura 2. El número de individuos adultos (a) y la extensión de ocurrencia (EOO) (b) son factores que determinan la vulnerabilidad de las 44 aves prioritarias asociadas con Polylepis ante el cambio climático. Sobre la base de los datos de la IUCN 2017 obtuvimos que el promedio y los rangos estimados de número de adultos de $\mathrm{n}$ especies (n) disminuyen con la categoría de amenaza (a), al igual que la media de la extensión de ocurrencia (b). Las barras en b muestran el intervalo de confianza del 95\% de la media (basado en la DS).

Figure 2. The number of mature individuals (a) and the extent of occurrence (EOO) (b) are factors that determine the vulnerability to climate change of the 44 conservation priority Polylepis birds. Based on IUCN 2017 data, we found that the estimated means and ranges the adult population of $\mathrm{n}$ species (n) decrease with increasing threat category (a), same as the mean EOO (b). The bars in b show the $95 \%$ confidence intervals of the mean (based on SD). 
en su totalidad, es importante reconocer que podrían desempeñar un papel clave en la conectividad del paisaje (Lloyd and Marsden 2008, 2011; Bellis et al. 2015; Sevillano-Ríos et al. 2011; Sevillano-Ríos and Rodewald 2017), por lo que debería promoverse su restauración y conservación. Esta idea también se puede asumir para otras especies leñosas de la zona como Gynoxys y Buddleja (Tinoco et al. 2013); 4) para favorecer la recuperación y la salud de los bosques remanentes es importante favorecer la regeneración natural del bosque (Morales et al. 2018). En este sentido, es clave reducir los niveles de fuego, pastoreo de ganado y tala (Cierjacks et al. 2008; Torres et al. 2008; Renison et al. 2010; Domic et al. 2014; Morales 2017). Incorporar a las comunidades humanas locales en estos esfuerzos es fundamental para alcanzar los objetivos de conservación en el largo plazo (e.g., Aucca and Ramsay 2005; Fuentealba and Sevillano-Ríos 2016), y 5) incorporar el cambio climático en los planes y estrategias de conservación de los ecosistemas de Polylepis a lo largo de los distintos países de su distribución. El sistema legal debería de proteger estos ecosistemas más allá de las áreas naturales protegidas, para lo cual, si se ven afectados, es necesario desarrollar mecanismos de medidas de mitigación y compensación. Organizaciones como la FAO, CONDESAN, UNESCO y el IPCC, entre otros, elaboraron publicaciones y documentos de trabajo para estos fines. En su mayoría, esta documentación es de libre acceso y disponibilidad.

Agradecimientos. Este trabajo se desarrolló como parte de los estudios de Maestría/PhD de CSSR, gracias al apoyo de Fulbright Schollarship y el Athena Grant 2014 y 2015. Agradecemos al Cornell Lab of Ornithology, al Kramer Graduate Fellowship Endowment y al E. Alexander Bergstrom Memorial Research Award 2005 de la Association of Field Ornithologists. También agradecemos al financiamiento de Cienciactiva, iniciativa del Consejo Nacional de Ciencia, Tecnología e Innovación Tecnológica (CONCYTEC); Contrato (237)- (2015)-FONDECYT. Un agradecimiento especial a M. Plenge por proporcionarnos artículos, a N. Young por su apoyo con la sistematización bibliográfica. Finalmente, queremos agradecer a D. Renison, E. Cuyckens, S. Morreale, V. Ruíz-Gutiérrez, E. Iñigo-Elias y a dos revisores anónimos que con sus comentarios y sugerencias ayudaron a mejorar el manuscrito.

\section{REFERENCIAS}

Alinari, J., A. von Müller, and D. Renison. 2015. The contribution of fire damage to restricting high mountain Polylepis australis forests to ravines: Insights from an un-replicated comparison. Ecología Austral 25:11-18.

Arnal, H., A. Sampson, G. Navarro, W. Palomino, W. Ferreira, K. Romoleroux, D. Caro, I. Teich, E. Cuyckens, C. Antezana, S. Arrazola, C. Aucca, J. Balderrama, S. Beck, S. Burneo, N. De la Barra, A. Bustamante, Y. Fandinso, G. Ferro, I. Gomez, G. Guzman, J. Iglesias, J. Irazabal, P. Lozano, M. Mercado, A. Monsalve, D. Renison, S. Salgado, and E. Samochuallpa. 2014. Mapa Pan Andino de Bosques de Polylepis prioritarios para Conservación. American Bird Conservancy, The Plains, USA.

Aucca, C., F. Olmos, O. Santander, and A. Chamorro. 2015. Range extension and new habitat for the Critically Endangered Royal Cinclodes Cinclodes aricomae. Cotinga 37:12-17.

Aucca, C., and P. M. Ramsay. 2005. Management of biodiversity and land use in southern Peru: ECOAN's activities to help conserve Polylepis woodlands. Mountain Research and Development 25:287-289.

Bellis, L. M., L. Rivera, N. Politi, E. Martin, M. L. Perasso, F. Cornell, and D. Renison. 2009. Latitudinal patterns of bird richness, diversity and abundance in Polylepis australis mountain forest of Argentina. Bird Conservation International 19:265-276.

Bellis, L. M., A. Pidgeon, C. Alcántara, S. Dardanelli, and V. Radeloff. 2015. Influences of succession and erosion on bird communities in a South American highland wooded landscape. Forest Ecology and Management 349:85-93. doi:10.1016/j.foreco.2015.03.047.

BirdLife International. 2017. IUCN Red List for birds. URL:www.birdlife.org (24/04/2017).

Cahill, J., and E. Matthysen. 2007. Habitat use by two specialist birds in high-Andean Polylepis forests. Biology Conservation 140:62-69.

Cierjacks, A., S. Salgado, K. Wesche, and I. Hensen. 2008. Post-fire population dynamics of two tree species in highaltitude Polylepis forests of Central Ecuador. Biotropica 40:176-182.

Colwell, R. K., G. Brehm, C. L. Cardelús, A. C. Gilman, and J. T. Longino. 2008. Global warming, elevational range shifts, and lowland biotic attrition in the wet tropics. Science 322:258-261.

Crick, H. Q. 2004. The impact of climate change on birds. Ibis 146:48-56.

Cuyckens, E., D. A. Christie, A. I. Domic, L.R.Malizia, and D. Renison. 2016. Climate change and the distribution and conservation of the world's highest elevation woodlands in the South American Altiplano. Glob Planet Change 137: 79-87.

Domic, A. I., G.R. Camilo, and J.M. Capriles. 2014. Small-scale farming and grazing reduce regeneration of Polylepis tomentella (Rosaceae) in the Semiarid Andes of Bolivia. Biotropica 46:106-114. doi: 10.1111/btp.12075. 
Fandiño, Y., and D. Caro. 2009. Mapa general de identificación de áreas potenciales de bosques de Polylepis en los Andes colombianos. Conservación Colombiana 10:7-44

Fjeldså, J. 1992. Biogeography of the birds of the Polylepis woodlands of the Andes. Pp. 71-84 in J. L. Luteyn and H. Balslev (eds.). Paramo, an Andean ecosystem under human influence. Academic Press, London, UK.

Fjeldså, J. 1993. The avifauna of the Polylepis woodlands of the Andean highlands: the efficiency of basing conservation priorities on patterns of endemism. Bird Conservation International 3: 37-55.

Fjeldså, J. 2002. Polylepis Forest-Vestiges of a vanishing Andean Ecosystem. Ecotropica 8:111-123.

Fjeldså, J., and N. K. Krabbe. 1990. Birds of the high Andes. Zoological Museum University of Copenhagen and Apollo Books, Copenhagen, Denmark.

Fjeldså, J., E. Lambin, and B. Mertens. 1999. Correlation between endemism and local ecoclimatic stability documented by comparing Andean bird distributions and remotely sensed land surface data. Ecography 22:63-78.

Fjeldså, J., R. Bowie, and C. Rahbek. 2012. The role of mountain ranges in the diversity of birds. Annu Rev Ecol Evol Syst 43:249-65.

Fraser, B. 2012. Melting in the Andes: Goodbye glaciers. Nature 491:180-182.

Frimer, O., and S. M. Nielsen. 1989. The status of Polylepis forests and their avifauna in Cordillera Blanca, Peru: technical report from an inventory in 1988, with suggestions for conservation management. Zoological Museum, University of Copenhagen, Copenhagen, Denmark.

Fuentealba, B., and C. S. Sevillano-Ríos. 2016. Experiencias de rehabilitación comunitaria con queñual (Polylepis sp.) en el Departamento de Ancash, Perú. Pp. 331 en E. Ceccon and D. R. Pérez (eds.). Más allá de la ecología de la restauración: perspectivas sociales en América Latina y el Caribe. Vázquez Mazzini Editores, Buenos Aires, Argentina.

Gareca, E. E., M. Hermy, J. Fjeldså, and O. Honnay. 2010. Polylepis woodland remnants as biodiversity islands in the Bolivian high Andes. Biodiversity and conservation 19:3327-3346.

GBIF.org. 2017. GBIF Occurrence. doi:10.15468/dl.v9sm0j.

Gosling, W. D., J. A. Hanselman, C. Knox, B. G. Valencia, and M. B. Bush. 2009. Long-term drivers of change in Polylepis woodland distribution in the central Andes. Journal of Vegetation Science 20: 1041-1052.

Herzog, S. K., R. Martínez, P. M. Jørgensen, and H. Tiessen. 2011. Climate change and biodiversity in the tropical Andes. São José dos Campos: Inter-American Institute for Global Change Research. Pp. 348.

Herzog, S. K., R. Soria, and E. Matthysen. 2003. Seasonal variation in avian community composition in a high-Andean Polylepis (Rosaceae) forest fragment. The Wilson Bulletin of Ornithology 115:438-447.

Hjarsen, T. 1999. Bird diversity and community structure. En Monitoring and management of high Andean biodiversity - a study from Cochabamba, Bolivia. Centre for Research on the Cultural and Biological Diversity of Andean Rainforests.

Hoch, G., and C. Körner. 2005. Growth, demography and carbon relations of Polylepis trees at the world's highest treeline. Functional Ecology 19:941-951.

Jameson, J. S., and P. M. Ramsay. 2007. Changes in high-altitude Polylepis forest cover and quality in the Cordillera de Vilcanota, Perú, 1956-2005. Biological Conservation 138:38-46.

Kessler, M. 1995. The genus Polylepis (Rosaceae) in Bolivia. Candollea 50:131-171.

Kessler, M. 2002. The "Polylepis problem": where do we stand. Ecotropica 8:97-110.

Kessler, M. 2006. Bosques de Polylepis. Pp. 110-120 en M. Baslev (eds.). Botánica económica de los Andes Centrales. Universidad Mayor de San Andrés, La Paz, Bolivia.

Kessler, M., and A. N. Schmidt-Lebuhn. 2006. Taxonomical and distributional notes on Polylepis (Rosaceae). Organisms diversity and evolution 6:67-69.

Kessler, M., J. M. Toivonen, S. P. Sylvester, J. Kluge, and D. Hertel. 2014. Elevational patterns of Polylepis tree height (Rosaceae) in the high Andes of Peru: role of human impact and climatic conditions. Frontiers in Plant Science 5:194. doi: $10.3389 /$ fpls.2014.00194.

La Sorte, F. A., and W. Jetz. 2010. Projected range contractions of montane biodiversity under global warming. Proceedings of the Royal Society of London B. Biological Sciences. doi: 10.1098/rspb.2010.0612.

Lloyd, H. 2008a. Abundance and patterns of rarity of Polylepis birds in the Cordillera Vilcanota southern Peru: implications for habitat management strategies. Bird Conservation International 18:164-180.

Lloyd, H. 2008b. Foraging ecology of high Andean insectivorous birds in remnant Polylepis forest patches. The Wilson Bulletin of Ornithology 120:531-544.

Lloyd, H. 2008c. Influence of within patch habitat quality on high Andean Polylepis bird abundance. Ibis 150:735745.

Lloyd, H., and S. J. Marsden. 2008. Bird community variation across Polylepis woodland fragments and matrix habitats: implications for biodiversity conservation within a high Andean landscape. Biodiversity and Conservation 17:26452660.

Lloyd, H., and S. J. Marsden. 2011. Between patch bird movements within a high Andean Polylepis woodland/matrix landscape: Implications for habitat restoration. Restoration Ecology 19:74-82.

Márcora, P., I. Hensen, D. Renison, P. Seltmann, and K. Wesche. 2008. The performance of Polylepis australis trees along their entire altitudinal range: implications of climate change for their conservation. Diversity and Distributions 144: 630-636.

Martínez, R., D. Ruiz, M. Andrade, L. Blacutt, D. Pabón, E. Jaimes, G. León, M. Villacís, J. Quintana, E. Montealegre, 
and C. Euscátegui. 2011. Synthesis of the climate of the Tropical Andes. S. K. Herzog, R. Martínez, P. M. Jørgensen and H. Tiessen (eds.). Climate change and biodiversity in the tropical Andes. São José dos Campos: Inter-American Institute for Global Change Research.

Matthysen, E., F. Collet, and J. Cahill. 2008. Mixed flock composition and foraging behavior of insectivorous birds in undisturbed and disturbed fragments of high-Andean Polylepis woodland. Ornitologia Neotropical 19:403-416.

Mazar-Barnett, J., G. D. Pugnali, and M. Della Seta. 1998. Primer registro del Sai grande Oreomanes fraseri (Passeriformes: Coerebidae, Thraupidae) en la Argentina. El Hornero 15:044-046.

Mendoza, W., and A. Cano. 2011. Diversidad del género Polylepis (Rosaceae, Sanguisorbeae) en los Andes peruanos. Revista Peruana de Biología 18:197-200.

Mendoza, W., and A. Cano. 2012. El Género Polylepis en el Perú. Taxonomía, Morfología y Distribución. Perú: Editorial Académica Española.

Møller, A., W. Fiedler, and P. Berthold. 2010. Effects of climate change on Birds. Oxford University Press. Pp. 321.

Morales, L. 2017. Polylepis regeneration and the potential for forest expansion in the Peruvian Andes: The influence of cattle and environmental conditions. Doctoral Dissertation, University of California Davis.

Morales, L.V., C. S. Sevillano., S. Fick., T. Young. 2018. Differential seedling regeneration patterns across forest-grassland ecotones in two tropical treeline species (Polylepis spp.). Austral Ecology. Early View. doi:/10.1111/aec.12588

Neelin, J. D., M. Münnich, H. Su, J. Meyerson, and C. Holloway. 2006. Tropical drying trends in global warming models and observations. Proceedings of the National Academy of Sciences 103:6110-6115.

Ocampo-Peñuela, N., C. N. Jenkins, V. Vijay, B. V. Li, and S. L. Pimm. 2016. Incorporating explicit geospatial data shows more species at risk of extinction than the current Red List. Sci Adv 2:e1601367.

Parker, T. A. III. 1981. Distribution and biology of the White-cheeked Cotinga Zaratornis stresemanni, a high Andean frugivore. Bulletin of the British Ornithologists' Club 101:156-265.

Parmesan, C. 2006. Ecological and evolutionary responses to recent climate change. Annual Review in Ecology Evolutions and Systematics. 37:637-669.

Purcell, J., and A. Brelsford. 2004. Reassessing the causes of decline of Polylepis, a tropical subalpine forest. Ecotropica 10:155-158.

Rabatel, A., B. Francou, A. Soruco, J. Gómez, B. Cáceres, J. L. Ceballos, R. Basantes, M. Vuille, J. Sicart, C. Huggel, M. Scheel, Y, Lejeune, Y. Arnaud, M. Collet, T. Condom, G. Consoli, V. Favier, V. Jomelli, R. Galarraga, P. Ginot, L. Maisincho, J. Mendoza, M. Menegoz, E. Ramírez, P. Ribstein, W. Suarez, M. Villacis, and P. Wagnon. 2013. Current state of glaciers in the tropical Andes: a multi-century perspective on glacier evolution and climate change. Cryosphere 7:81-102.

Rehm, E., and K. Feely. 2013. Forest patches and the upward migration of timberline in the southern Peruvian Andes. Forest Ecology and Management 305:204-211.

Rehm, E., and K. Feeley. 2016. Many species risk mountain top extinction long before they reach the top. Frontiers of Biogeography 8:e27788.

Renison, D., I. Hensen, and A. M. Cingolani. 2006. Cover and growth habit of Polylepis woodlands and shrublands in the mountains of central Argentina: human or environmental influence? Journal of Biogeography 33:876-887.

Renison, D., I. Hensen, R. Suárez, A. M. Cingolani, P. Márcora, and M. A. Giorgis. 2010. Soil conservation in Polylepis mountain forests of Central Argentina: Is livestock reducing our natural capital? Austral Ecology 35:435-443.

Renison, D., G. A. Cuyckens, S. Pacheco, G. F. Guzmán, H. R. Grau, P. Márcora, G. Robledo, A. M. Cingolani, J. Domínguez, M. Landi, and L. Bellis. 2013. Distribución y estado de conservación de las poblaciones de árboles y arbustos del género Polylepis (Rosaceae) en las montañas de Argentina. Ecología Austral 23:27-36.

Renison, D., M. L. Herrero, R. Torres, R. Suárez, P. Friedlander, S. Navarro, F. Barri, and A. Cingolani. 2016. El rol de los voluntariados en la restauración ecológica del centro argentino. Pp. 55-76 en E. Ceccon and D. R. Pérez (eds.). Más allá de la ecología de la restauración: perspectivas sociales en América Latina y el Caribe. Vázquez Mazzini Editores, Buenos Aires, Argentina.

Ridbäck, U. A. 2008. Floristic Study of Polylepis Forest Fragments in the Central Andes of Ecuador. 25. Gotland University, Visby, Sweden.

Robledo, G., and D. Renison. 2010. Wood-decaying polypores in the mountains of central Argentina in relation to Polylepis forest structure and altitude. Fungal Ecology 3:178-184

Schulenberg, T. S., D. F. Stotz, D. F. Lane, J. P. O’Neill, and T. A. Parker III. 2010. Birds of Peru: revised and updated edition. Princeton University Press, Princeton, New Jersey.

Şekercioğlu, Ç. H., S. H. Schneider, J. P. Fay, and S. R. Loarie. 2008. Climate change, elevational range shifts, and bird extinctions. Conservation Biology 22:140-150.

Şekercioğlu, Ç. H., R. B. Primack, and J. Wormworth. 2012. The effects of climate change on tropical birds. Biology and Conservation 1481:1-18.

Servat, G. P. 2006. The role of local and regional factors in the foraging ecology of birds associated with Polylepis woodlands. Doctoral Dissertation, University of Missouri-St. Louis, USA. Dissertations 588. URL: goo.gl/Umxhkt.

Sevillano-Ríos, C. S. 2010. Efectos de la fragmentación y degradación de hábitat de queñual (Polylepis spp.) con respecto a la avifauna asociada en la Reserva de Biosfera Huascarán. Tesis de Licenciatura, Universidad Peruana Cayetano Heredia. Lima. Perú. Pp. 102.

Sevillano-Ríos, C. S. 2016. Diversity ecology and conservation of bird communities of Polylepis woodlands in the 
northern Andes of Peru. Master Thesis, Cornell University. Ithaca. USA. Pp. 149.

Sevillano-Ríos, C. S., H. Lloyd, and A. Valdés-Velásquez. 2011. Bird species richness diversity and abundance in Polylepis woodlands Huascaran Biosphere Reserve Peru. Studies on Neotropical Fauna and Environment 46:69-76.

Sevillano-Ríos, C. S., and A. D. Rodewald. 2017. Avian community structure and habitat use of Polylepis forests along an elevation gradient. PeerJ 5:e3220. doi: 10.7717/peerj.3220.

Simpson, B. B. 1979. A revision of the genus Polylepis (Rosaceae). Sanguisorbeae No. 43. Smithsonian Institution Press, Washington, DC.

Sylvester, S. P., M. D. Sylvester, and M. Kessler. 2014. Inaccessible ledges as refuges for the natural vegetation of the high Andes. Journal of Vegetation Science 25:1225-1234.

Tinoco, B. A., P. X. Astudillo, S. C. Latta, D. Strubbe, and C. H. Graham. 2013. Influence of patch factors and connectivity on the avifauna of fragmented Polylepis forest in the Ecuadorian Andes. Biotropica 45:602-611.

Toivonen, J. M. 2014. Determinants of Polylepis (Rosaceae) forest distribution and treeline formation in the high Andes. Doctoral thesis. University of Turku. Finlandia. Pp. 41.

Torres, R. C., D. Renison, I. Hensen, R. Suárez, and L. Enrico. 2008. Polylepis australis' regeneration niche in relation to seed dispersal, site characteristics and livestock density. Forest Ecology and Management 254:255-260.

Urrutia, R., and M. Vuille. 2009. Climate change projections for the tropical Andes using a regional climate model: Temperature and precipitation simulations for the end of the 21st century. Journal of Geophysical Research 114: D02108. doi: 10.1029/2008JD011021.

Valderrama, S., and J. C. Verhelst. 2009. Avifauna asociada a los bosques de Polylepis en Colombia. Conservación Colombiana 10:45-68.

Valencia, B. G., M. B. Bush., A. L. Coe., E. Orren., and W. D. Gosling. 2018. Polylepis woodland dynamics during the last 20,000 years. J Biogeogr. 00:1-12. https://doi.org/10.1111/jbi.13209

Vuille, M., B. Francou, P. Wagnon, I. Juen, G. Kaser, B. G. Mark, and R. S. Bradley. 2008. Climate change and tropical Andean glaciers: Past present and future. Earth-Sci Rev 89:79-96.

Vuilleumier, F. 1984. Patchy distribution and systematics of Oreomanes fraseri (Aves, Coerebidae) of Andean Polylepis woodlands. American Museum Novitates 2777:1-17.

Williams, R., and J. Ferrigno. 1999. Glaciers of South America. In R. Williams and J. Ferrigno (eds.). Satellite Image Atlas of Glaciers of the World. U.S. Geological Survey professional paper 1386. ISBN 0-607-92475-6. URL: pubs.usgs.gov/ $\mathrm{pp} / \mathrm{p} 1386 \mathrm{i}$.

Wormworth, J., and C. H. Sekercioglu. 2011. Winged sentinels: birds and climate change. Cambridge University Press. Pp. 262.

Wormworth, J., and K. Mallon. 2007. Bird Species and Climate Change. The Global Status Report. Climate Risk, New South Wales (Australia). Pp. 75.

Yallico, E. 1992. Distribución de Polylepis en el sur de Puno. Arbolandino. Puno, Perú. Pp. 134.

Young, K. 2011. Introduction to Andean Geographies. Pp. 128-140 in S. K. Herzog, R. Martínez, P. M. Jørgensen and H. Tiessen (eds.). Climate Change and Biodiversity in the Tropical Andes. Inter-American Institute for Global Change Research (IAI) and Scientific Committee on Problems of the Environment (SCOPE). ISBN: 978-85-99875-05-6. doi: 10.13140/2.1.3718.4969.

Zutta, B. R., P. W. Rundel, S. Saatchi, J. D. Casana, P. Gauthier, A. Soto, and W. Buermann. 2012. Prediciendo la distribución de Polylepis: bosques Andinos vulnerables y cada vez más importantes. Revista Peruana de Biología 19:205-212. 